\title{
Corrigendum: Spatial Continuity Effect vs. Spatial Contiguity Failure. Revising the Effects of Spatial Proximity Between Related and Unrelated Representations
}

\author{
Maik Beege ${ }^{1 *}$, Maria Wirzberger ${ }^{2}$, Steve Nebel ${ }^{1}$, Sascha Schneider ${ }^{1}$, Nina Schmidt ${ }^{1}$ and \\ Günter Daniel Rey ${ }^{1}$
}

${ }^{1}$ Faculty of Humanities, Technische Universität Chemnitz, Chemnitz, Germany, ${ }^{2}$ Max Planck Research Group "Rationality Enhancement", Max Planck Institute for Intelligent Systems, Tübingen, Germany

Keywords: split-attention effect, proximity, integration, spatial contiguity principle, spatial contiguity failure

\section{OPEN ACCESS}

Edited and reviewed by: Monika Akbar

The University of Texas at El Paso,

United States

*Correspondence: Maik Beege

maik.beege@phil.tu-chemnitz.de

Specialty section:

This article was submitted to Digital Education,

a section of the journal

Frontiers in Education

Received: 09 March 2020 Accepted: 06 April 2020 Published: 28 April 2020

Citation:

Beege M, Wirzberger M, Nebel S, Schneider S, Schmidt N and Rey GD (2020) Corrigendum: Spatial Continuity Effect vs. Spatial Contiguity Failure. Revising the Effects of Spatial Proximity Between Related and Unrelated Representations. Front. Educ. 5:46. doi: 10.3389/feduc.2020.00046

\section{A Corrigendum on}

Spatial Continuity Effect vs. Spatial Contiguity Failure. Revising the Effects of Spatial Proximity Between Related and Unrelated Representations

by Beege, M., Wirzberger, M., Nebel, S., Schneider, S., Schmidt, N., and Rey, G. D. (2019). Front. Educ. 4:86. doi: 10.3389/feduc.2019.00086

In the original article, there was an error. By describing the results of experiment 1 with regard to the learning outcomes, we accidentally reported incorrect statistical data. We reported the correct conclusions in the results part (non-significant) but the data did not match this statement. The error occurred because we copied the data from another section and forgot to change the statistical values.

A correction has been made to the Experiment 1 section, subsection Results, sub-subsection Learning, paragraph 2:

"With respect to H1a, follow-up contrast analyses (high proximity: $\lambda=1$; medium proximity: $\lambda=1$; low proximity: $\lambda=1$; separated condition: $\lambda=-3$ ) were conducted in order to test our postulated results pattern. In terms of retention, the results pattern could not be supported $t=$ $0.66, S E=0.26, p=0.51, r=0.07$. In terms of transfer, the contrast analysis also revealed a nonsignificant result $(t=1.47, S E=0.15, p=0.15, r=0.15)$. The separated condition did not generally score lower than the spatially integrated conditions."

The authors apologize for this error and state that this does not change the scientific conclusions of the article in any way. The original article has been updated.

Copyright $\odot 2020$ Beege, Wirzberger, Nebel, Schneider, Schmidt and Rey. This is an open-access article distributed under the terms of the Creative Commons Attribution License (CC BY). The use, distribution or reproduction in other forums is permitted, provided the original author(s) and the copyright owner(s) are credited and that the original publication in this journal is cited, in accordance with accepted academic practice. No use, distribution or reproduction is permitted which does not comply with these terms. 